\title{
Heterogeneous reactions to heterogeneity in returns from public goods
}

\author{
Urs Fischbacher Simeon Schudy \\ Sabrina Teyssier
}

\begin{abstract}
In many cases individuals benefit differently from the provision of a public good. We study in a laboratory experiment how heterogeneity in returns and uncertainty about the own return affects unconditional and conditional contribution behavior in a linear public goods game. The elicitation of conditional contributions in combination with a within subject design allows us to investigate belief-independent and type-specific reactions to heterogeneity. We find that, on average, heterogeneity in returns decreases unconditional contributions but affects contributions only weakly. Uncertainty in addition to heterogeneity reduces conditional contributions slightly. Individual reactions to heterogeneity differ systematically. Selfish subjects and one third of conditional cooperators do not react to heterogeneity whereas the reactions of the remaining conditional cooperators vary. A substantial part of heterogeneity in reactions can be explained by inequity aversion with respect to different reference groups.
\end{abstract}

\author{
U. Fischbacher $\cdot$ S. Schudy \\ University of Konstanz and Thurgau Institute of Economics, \\ Post Box 131, 78457 Constance, Germany \\ e-mail: Urs.Fischbacher@uni-konstanz.de \\ S. Schudy \\ e-mail: Simeon.Schudy@uni-konstanz.de \\ S. Teyssier $(\varangle)$ \\ INRA, UR1303 ALISS, 94200 Ivry-sur-Seine, France \\ e-mail: Sabrina.Teyssier@ivry.inra.fr
}




\section{Introduction}

Investments in public goods (e.g. investments in energy-saving measures) benefit the investor and others. The value of obtained benefits (e.g. individual cost savings, reduction in $\mathrm{CO}_{2}$ emissions or clean air) is in many cases difficult to assert and different individuals benefit differently from the public good. In order to develop policies to sustain the provision of public goods, it is thus crucial to understand how uncertainty and heterogeneity in returns from public goods affect contribution behavior. Previous experimental work has focused on aggregate effects of heterogeneous returns from public goods on people's unconditional contributions to public goods (see e.g. Fisher et al. 1995) and uncertainty of returns (see e.g. Dickinson 1998, and Levati et al. 2009). However, unconditional contributions depend on beliefs about others' contributions whereas the analysis of conditional contributions allows to control the effect of beliefs. Further, if people are heterogeneous in their preferences or in their reference points (i.e. they compare to different reference groups), their reactions to heterogeneity will differ in systematic ways. Studying aggregate effects may then lead to wrong conclusions and entail wrong policy implications. The aim of our paper is therefore to focus on belief-independent and type-specific reactions to heterogeneity.

The novelty of our experimental design is twofold: First, on top of unconditional contributions we elicit conditional contributions of subjects and thereby isolate beliefindependent reactions to heterogeneity. Second, we use a within-subject design which allows us to identify type specific reactions to heterogeneity. Additionally, we provide insights on how people perceive heterogeneity in returns by relating our results to theoretical predictions based on two social preference models which we extend to allow for different reference groups to which people may compare.

In the experiment participants play several one-shot linear public goods games in groups of four. The social return from the public good is identical in all the games but we vary the marginal per capita returns (MPCRs). Subjects make unconditional and conditional contributions with certain and homogeneous MPCRs, certain and heterogeneous MPCRs and uncertain and heterogeneous MPCRs. In each game with heterogeneity in MPCRs, two group members receive a high MPCR while the two others receive a low MPCR. Uncertainty only concerns subjects' own MPCRs whereas the distribution of MPCRs is always known.

We find that unconditional contributions are negatively affected by the introduction of heterogeneity in MPCRs from the public good. Conditional contributions are however not significantly affected by heterogeneity. This indicates that negative effects of heterogeneity on contributions to public goods mainly stem from pessimistic beliefs about other's contributions. In heterogeneous environments, uncertainty about the own MPCR does not decrease unconditional contributions further and affects conditional contributions only weakly. Further we show that individual reactions to heterogeneity differ systematically. Selfish subjects and one third of conditional cooperators do not modify their conditional contributions to the public good when heterogeneity in returns is introduced. Around $17 \%$ of conditional cooperators increase contributions when receiving the high return and decrease contributions when receiving the low return. Additionally, we observe that $27 \%$ of conditional cooperators react only to 
either high or low MPCRs. Another $25 \%$ of conditional cooperators show the same reaction (an increase or a decrease) regarding both returns.

Since the early experiments reported in Bohm (1972), a vast experimental literature on public goods has grown, showing that individuals invest in public goods even though the individual marginal return from investments to the public good is lower than the individual marginal cost. ${ }^{1}$ Because contributions vary with the own returns from the public good [see e.g. Ledyard (1995), heterogeneity in returns may affect contribution behavior]. An early experiment by Fisher et al. (1995) focused on the comparison of contributions to a public good by subjects with the same MPCR under homogeneity and heterogeneity in MPCRs. They neither find strong support for socalled "seeding" (i.e. higher contributions by subjects with low MPCRs in case of heterogeneity in MPCRs) nor for a "poisoning of the well" (i.e. lower contributions by subjects with high MPCRs in case of heterogeneity in MPCRs). However, in their experiment, subjects were only told that heterogeneity in returns is possible. Subjects did not know whether returns were actually different. Other experimental studies indicate that heterogeneous valuations of the public good lead less frequently to the efficient outcome [see e.g. Marwell and Ames (1980), Bagnoli et al. (1991), Chan et al. (1999), Carpenter et al. (2009), Reuben and Riedl (2009), Nikiforakis et al. (2012).${ }^{2}$ However, these studies do not elicit conditional contributions and thus cannot disentangle whether the decrease in average contributions is due to pessimistic beliefs about other group members" contributions or due to "pure" inequity considerations. Our experimental design allows us to go beyond this limitation. In particular, the data indicate that heterogeneity matters for unconditional but not necessarily for conditional contributions and thus suggest that heterogeneity primarily affects beliefs about others' contributions.

Heterogeneity in returns is also closely related to uncertainty about returns because the latter involves different possible returns by construction. Dickinson (1998) and Levati et al. (2009) study the effects of uncertainty in MPCRs and find significantly lower unconditional contributions when the MPCR is stochastic compared to a certain return. Gangadharan and Nemes (2009) differentiate between situations in which the probabilities for low and high MPCRs are known by the subjects and situations with unknown probabilities. In both situations, unconditional contributions are significantly lower when there is uncertainty in the returns compared to a certain homogeneous return. However, these studies do not separate the effects of uncertainty from the effects of heterogeneity in returns additionally to the fact that they only analyze unconditional contributions. We isolate the effect of uncertainty about the own MPCR by comparing unconditional and conditional contributions to the public good when there is heterogeneity in returns and the own returns are known with contributions when there is heterogeneity in returns but own returns are uncertain.

\footnotetext{
1 See e.g. Ledyard (1995), Anderson (2001), Gächter (2007) or Chaudhuri (2011) for surveys.

2 Note that we only consider heterogeneity in valuations of public goods. For heterogeneity in productivity see e.g. Tan (2008) or Fellner et al. (2011) and for heterogeneity in valuations of the private good see e.g. Falkinger et al. (2000). For a meta study on determinants of contributions in linear public goods games see Zelmer (2003). Her findings indicate that heterogeneity decreases contributions; strongly for endowment heterogeneity and weakly for heterogeneity in MPCRs.
} 
Our results provide insights for behavioral theories and political action. With respect to behavioral theories, we show that subjects react differently to the introduction of heterogeneity in returns. Reactions to heterogeneity are in line with the idea that conditional cooperators refer to different reference groups when deciding about contributing to the public good. Consequently, developing reciprocity or inequality aversion models which take differences in reference groups into account seems a promising research plan. As heterogeneity in returns from public goods reduces unconditional contributions but affects conditional contributions only slightly, policy implications are twofold. First, politics may aim at reducing heterogeneity in returns through compensations. Second, communication policies which counteract pessimistic beliefs about others' contributions may be effective.

The remainder of this paper is organized as follows. In Sect. 2 we present the experimental design. In Sect. 3 we propose theoretical predictions and highlight the importance of subjects' reference group. Section 4 presents the results of the experiment and Sect. 5 concludes.

\section{Experimental design and procedures}

Subjects played six different versions of a standard one-shot linear public goods game in groups of four. At the beginning of the experiment we informed subjects that they would participate in several experiments, but we did not inform them in advance about the specific features of the six versions of the linear public goods game. Also subjects were informed that one of the games played would be randomly selected to be payoff relevant at the end of the experiment. ${ }^{3}$ Because we distributed the instructions for each game just before the game started, subjects' decisions in each public good game did not depend on any of the characteristics of the subsequent public good games. Subjects received feedback only after the last game and were informed about this at the beginning of the experiment. In all six games, subjects received an endowment of 20 points each and the monetary payoff function was the following:

$$
y_{i}=20-g_{i}+\gamma_{i} \sum_{j=1}^{4} g_{j}
$$

with $y_{i}$ representing subject i's monetary income, $g_{i}$ denoting i's contribution to the public good, and $\gamma_{i}$ equal to the MPCR of an investment by subject $i$. In the first three public good games subjects made unconditional contribution decisions (UC games). In the second three public good games we elicited conditional contributions (CC games).

In treatment UC04, all group members received the same MPCR from the public good: $\gamma_{i}=0.4$. Each subject decided on her unconditional contribution and the game ended. In UCu0305, we introduced heterogeneity of MPCRs with uncertainty about each subject's own MPCR. Two subjects received $\gamma_{\mathrm{L}}=0.3$ and two subjects received $\gamma_{\mathrm{H}}=0.5$. When making their contribution decisions in UCu0305, subjects did not

\footnotetext{
3 We do not report results on a seventh decision (a donation decision) made by our subjects which was also elicited and included in the random selection of payoffs.
} 
know whether they would receive $\gamma_{\mathrm{L}}$ or $\gamma_{\mathrm{H}}$ but they did know that two subjects in the group would receive $\gamma_{\mathrm{L}}$ and two would receive $\gamma_{\mathrm{H}}$. Thus, in UCu0305 there is uncertainty about the own MPCR, but the distribution of MPCRs is known. Note further that the marginal social return from the public good is unchanged. In the third game, there is heterogeneity of MPCRs but no uncertainty about the own MPCR. Because we are interested in type specific reactions to heterogeneity, we needed to elicit the contribution behavior of each individual for both cases, facing $\gamma_{\mathrm{L}}$ and $\gamma_{\mathrm{H}}$. In order to keep the payoff relevance of decisions in the third game equivalent to the decisions in the homogeneous case and the heterogeneous case with uncertainty we used the strategy method to elicit contributions in the third game. That is subjects stated their contribution conditional on having the low (UC03) or high (UC05) MPCR, knowing that two subjects in the group would receive $\gamma_{\mathrm{L}}$ and two would receive $\gamma_{\mathrm{H}}$. Subjects were informed that at the end of the experiment a roll of a die would randomly select for which of the two group members the low (or high) MPCR was payoff relevant. Alternatively, we could have used two direct response method treatments, one in which one half of the subjects faced the low and one half faced the high MPCR and a second in which it was the other way around. Both elicitation methods involve some uncertainty about the payoff relevance of the decisions made (because of the random selection of the payoff relevant game at the end of the experiment) but exclude uncertainty about subjects' own MPCR. We decided to use the strategy method in order to easily guarantee the same relevance of decisions in the third game compared to the first two games.

In the three CC games, we elicited conditional contributions which do not depend on subjects' beliefs about the average contribution of their group members. We used the procedure introduced by Fischbacher et al. (2001) in order to elicit conditional contributions. The procedure uses a variant of the strategy method (Selten 1967). Subjects first decide on their unconditional contribution and then fill in a conditional contribution table. They state how many points they wish to contribute dependent on the average contribution of their group members' $\bar{g}$. ${ }^{4}$ For each group, a random device (a die) selects one subject for whom the conditional contribution is relevant and three subjects for whom the unconditional contribution is relevant. MPCRs and information about possible MPCRs are equivalent to the information in the UC games. Table 1 summarizes the treatments.

In all sessions, CC games were conducted after UC games to have a progression of complexity in games. However, we altered the order among UC and CC games to control for changes in subjects' contributions as the session progresses. In six sessions, the order was first UC04, then UCu0305 and finally UC03/UC05 (first homogeneity then heterogeneity) while in four sessions the order was UC03/UC05, UCu0305 and finally UC04 (first heterogeneity then homogeneity). The order in CC games followed the order in UC games. At the end of the session, three rolls of a die decided on which of the games was payoff relevant, which subject's contribution table would be implemented and which two subjects in a group faced the low and high MPCR in the games with heterogeneity. We computerized the experiment using z-Tree (Fischbacher

\footnotetext{
4 Averages are rounded to integer numbers, i.e. subjects have to fill in 21 values. The translated instructions in the online appendix provide a screenshot.
} 
Table 1 Treatments

\begin{tabular}{ll}
\hline Type of game and MPCR & Name \\
\hline Unconditional cooperation games (UC games) & \\
$\gamma_{i}=0.4$ & $\mathrm{UC0} 0$ \\
$\gamma_{i}=0.3$ or $\gamma_{i}=0.5$, with uncertainty & $\mathrm{UCu} 0305$ \\
$\gamma_{i}=0.3$ (with heterogeneity) & $\mathrm{UC03}$ \\
$\gamma_{i}=0.5$ (with heterogeneity) & $\mathrm{UC05}$ \\
Conditional cooperation games (CC games) & \\
$\gamma_{i}=0.4$ & $\mathrm{CC} 04$ \\
$\gamma_{i}=0.3$ or $\gamma_{i}=0.5$, with uncertainty & $\mathrm{CCu0305}$ \\
$\gamma_{i}=0.3$ (with heterogeneity) & $\mathrm{CC} 03$ \\
$\gamma_{i}=0.5$ (with heterogeneity) & $\mathrm{CC} 05$ \\
\hline
\end{tabular}

2007). Each subject sat at a randomly assigned and separated computer terminal and was given a copy of instructions. ${ }^{5}$ A set of control questions was provided to ensure the understanding of the game. If any participant repeatedly failed to answer correctly, the experimenter provided an oral explanation. No form of communication between the subjects was allowed during the experiment.

We conducted all sessions at the Lakelab (University of Konstanz, Germany). The data were collected over 10 sessions with 228 participants in total. The sessions took place between November 2009 and January 2010 and in February 2011. The experiment lasted about $1 \mathrm{~h}$ and $30 \mathrm{~min}$. Participants received on average 21.96 euros including a show-up fee of 4 euros. We recruited participants from the local subject pool including undergraduate and graduate students of all fields of studies (46\% male) using ORSEE Greiner (2004).

\section{Theoretical predictions}

Selfish subjects have a dominant strategy not to contribute in the UC games. In the CC games, the conditional cooperation of selfish subjects is also zero for all contribution levels of the other subjects. These predictions do not depend on our treatment variations. However, experimental research on public goods games has shown that people are willing to contribute significantly more to the public good than suggested by the assumption of selfishness. Several models have been suggested to explain such behavior: reciprocity models [e.g. Rabin (1993), Dufwenberg and Kirchsteiger (2004), and Falk and Fischbacher (2006)] or models of inequity aversion [e.g. Fehr and Schmidt (1999), and Bolton and Ockenfels (2000)]. All these theories predict some form of conditional cooperation if the players have a sufficiently strong social motive.

In this section, we discuss the theoretical predictions for conditional contributions by players with non-selfish preferences for our versions of the linear public goods game. We focus on conditional contribution behavior, because players' unconditional contributions in the UC games depend on players' beliefs about other players' contri-

\footnotetext{
5 A copy of translated instructions can be found in the online appendix.
} 
butions. In particular, we analyze how distributional-based concerns of players affect their behavior and then discuss predictions of two well known inequity aversion models by Fehr and Schmidt (1999), and Bolton and Ockenfels (2000). Additionally, we discuss how players should behave according to these models if they have specific reference groups to which they compare themselves. ${ }^{6}$

In the model of Fehr and Schmidt (1999) (from now on FS model), individuals maximize a utility function of the following type:

$$
U\left(y_{i}, y_{j}\right)=y_{i}-\frac{\alpha_{i}}{n-1} \sum_{j, y_{j}>y_{i}}\left(y_{j}-y_{i}\right)-\frac{\beta_{i}}{n-1} \sum_{j, y_{j}<y_{i}}\left(y_{i}-y_{j}\right)
$$

with $\alpha_{i} \geq \beta_{i}$ and $0 \leq \beta_{i}<1$. The parameter $\alpha_{i}$ represents individual i's disadvantageous inequity aversion (or envy) while $\beta_{i}$ corresponds to her advantageous inequity aversion. The monetary payoff of individual $i$ is $y_{i}$ as defined by Eq. (1). $y_{j}$ denotes the income of players $\mathrm{j}$. According to the FS model, subjects with a sufficiently high disutility from advantageous inequality $\left(\beta_{i}\right)$ are willing to contribute to the public good in order to reduce the advantageous inequality, given others contribute. Because of the linearity of the public good game and of the FS model, for all but a finite set of values of border case parameters the best reply is zero contribution, full contribution, or a contribution that generates equal payoffs with some player. In particular, if all players have the same MPCR, $\gamma=0.4$, then conditional cooperation is either zero or perfect (i.e. $g_{i}=\overline{\mathrm{g}}$ ): it is perfect for players with $\beta>0.60$.

In an equilibrium with positive contributions all payoffs have to be the same. Thus, in the heterogeneous case, players with high MPCRs have to contribute 7/3 as much as players with low MPCRs. ${ }^{7}$ Equilibria with positive contributions are thus characterized by the fact that players with an MPCR of 0.5 contribute $61.5 \%$ above the average of all other group members and players with the low MPCR $52.9 \%$ of the average contribution of all other group members). Consequently, conditional contributions of players with $\gamma_{i}=0.5$ (with $\gamma_{i}=0.3$ ) are higher (lower) than conditional contributions of players receiving $\gamma=0.4$ in the game with homogenous MPCRs. Therefore, heterogeneity in MPCRs will lead to higher average conditional contributions in $\mathrm{CC} 05$ than in CC04 than in CC03. Because of the fixed upper-bound of contributions, heterogeneity in MPCRs should lead (on average) to lower conditional contributions than in $\mathrm{CC} 04 .{ }^{8}$ If uncertainty is introduced, the FS model allows for

\footnotetext{
6 Note that in our experiment, subjects do not have explicit information about inequity in contributions of the other group members but only condition on the average contribution of their group members. Thus our study focuses on public goods for which people are aware that they may benefit equally or unequally but individual contributions are not observable (e.g. the water quality of a lake or air quality in a city). Results by Cheung (2011) and Wolff (2013) show that if information on individual contributions is known, higher variances in contributions will cause a reduction in conditional contributions.

7 Note that all members making the same contribution is not plausible in an equilibrium with positive contributions. With equal contributions, it is optimal for individuals with the high MPCR to contribute the same amount as the group average but for individuals with the low MPCR it is optimal to contribute 1/3 of the group average.

8 Besides, because the threshold for a contribution equalizing payoffs is lower for the player with the high MPCR and higher for those with the low MPCR than the threshold for the situation in which all individuals
} 


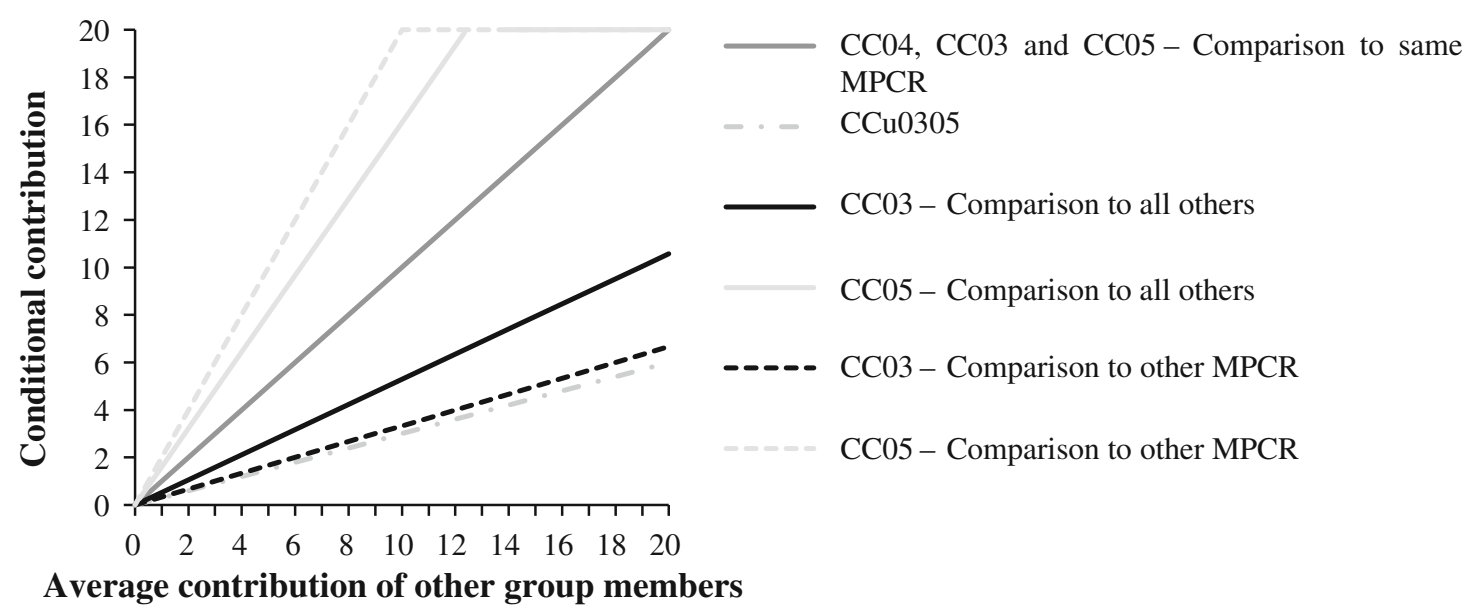

Fig. 1 Optimal conditional contributions (FS model, $\beta=0.8$ )

positive conditional contributions but predicts a strong decrease in total conditional contributions. Positive conditional contributions will be lower or equal to $35 \%$ of the group members' average contribution. The sharp drop in contributions results from the fact that "over-contributing" in the case of facing the low MPCR weights stronger than "under-contributing" when facing the high MPCR. Uncertainty will then make the average of conditional contributions lower than in the games without uncertainty with or without heterogeneity.

As a further benchmark, we extend the FS model by assuming that some players compare only to a specific reference group. Players who compare only to the counterpart who has the same MPCR contribute exactly the average contribution of their group members if their $\beta$ is sufficiently high. If players compare only with group members who have a different MPCR, players with $\gamma_{i}=0.3$ contribute $1 / 3$ of the average contributions whereas players with $\gamma_{i}=0.5$ contribute twice the average. 9 Consequently, optimal conditional contribution levels are different depending on the reference group of players: they are identical in $\mathrm{CC} 04$ and in $\mathrm{CC} 03$ and $\mathrm{CC} 05$ if players' reference group includes the player with the same MPCR whereas they are higher in $\mathrm{CC} 05$ than in $\mathrm{CC} 04$ and than in $\mathrm{CC} 03$ if players' reference group includes all other players or only players with the other MPCR. We show all predictions in Fig. 1 for $\beta=0.8$.

We now turn to the predictions of the model of equity, reciprocity and competition by Bolton and Ockenfels (2000) (from now on ERC model). In the ERC model it is assumed that each agent i maximizes the following utility function:

$$
U_{i}=U_{i}\left(y_{i}, \frac{y_{i}}{c}\right)
$$

Footnote 8 continued

face the same MPCR of 0.4, we should also observe more people contributing positive amounts in situation CC05 than in CC04, and more in CC04 than in CC03.

9 Note that the threshold is smaller for a player with the low MPCR than for an individual with the high MPCR because it is less costly for the player with the low MPCR to reduce inequality (he loses 0.7 by contributing a unit and each member of his reference group gains 0.5 whereas a player with the high MPCR loses 0.5 when contributing 1 unit while his reference group members gain only 0.3 each). 
The utility of each agent depends on her monetary payoff $y_{i}$ and her relative payoff $\frac{y_{i}}{c}$. The sum of all group members' monetary payoffs is represented by $c, c=\sum_{j=1}^{n} y_{i}$. Based on Bolton and Ockenfels (2000), in our framework each agent maximizes the following utility function:

$$
U_{i}=y_{i}-\vartheta_{i}\left(\frac{y_{i}}{y_{i}+3 \bar{y}}-\frac{1}{4}\right)^{2}
$$

with $\bar{y}$ being the average payoff of the other group members. The parameter $\vartheta_{i} \geq$ 0 , represents an individual preference parameter and expresses the importance of disutility from inequality. The higher $\vartheta_{i}$, the more inequity averse the subject i. If $\vartheta_{i}$ is sufficiently high, players will conditionally contribute to the public good. In the homogeneous case, conditional cooperation is weakly increasing in $\vartheta_{i}$ as well as in the own MPCR. If there is heterogeneity in MPCRs, it is not generally true that an increase in the own MPCR generates an increase of the own contribution. However, due to the discrete nature of the experiment, it can be shown numerically to hold for the parameters chosen in the experiment. Using a numerical approach we obtain weakly higher average conditional contributions and a steeper slope of contribution schedules in $\mathrm{CC} 05$ than in $\mathrm{CC} 04$ than in $\mathrm{CC} 03 .{ }^{10}$ Again, for sufficiently high values of $\vartheta_{i}$, heterogeneity in MPCRs should lead to lower average conditional contributions than in $\mathrm{CC} 04$ because of the fixed upper-bound of contributions. Using this procedure reveals also that according to the ERC model conditional contributions are weakly higher in $\mathrm{CC} 04$ than in $\mathrm{CCu} 0305$ but the difference amounts to at most one point. Conditional contributions in games with heterogeneity in MPCRs without uncertainty and with uncertainty should not differ strongly.

In order to understand systematic differences in reactions to heterogeneity, we extend also the ERC model by allowing subjects to differ in their reference group. Formally, we replace the value of $\bar{y}$ in (4) by the average payoff of the respective reference group. Figure 2 includes optimal conditional contributions for all games with certainty about the own MPCR. ${ }^{11}$ Optimal conditional contributions are shown for value $\vartheta_{i}=2000$ but the order of the conditional contribution schedules in Fig. 2 does not depend on the parameter value of $\vartheta_{i}$. The spread between conditional contributions in $\mathrm{CC} 03$ and $\mathrm{CC} 05$ will be larger if subjects compare their own payoff only to the average payoff of group members with the other MPCR than if they compare their payoff to all group members. Instead, they will roughly contribute the same in $\mathrm{CC} 03, \mathrm{CC} 04$ and $\mathrm{CC} 05$, if they compare their own payoff to the payoff of the other group member receiving the same MPCR. We briefly summarize the predictions of the FS model and of the ERC model in Table 2.

\footnotetext{
${ }^{10}$ Due to the discrete nature of the experiment only a finite set of contribution schedules exists. The logic of the numerical analysis works as follows. First, it can be shown that conditional contributions are monotonically increasing in for both the homogeneous and the heterogeneous case (with and without uncertainty about the own MPCR). Because this is the case, it is sufficient to show in a second step that the increase in $\vartheta_{\mathrm{i}}$ increases conditional contributions "step wise" by one unit across the treatment in the order shown such that the weak inequalities $\mathrm{CC} 05=\mathrm{CC} 04=\mathrm{CCun0305}=\mathrm{CC} 03$ always holds .

11 We do not include the optimal contributions for $\mathrm{CCu} 0305$, which are weakly below optimal contribution in $\mathrm{CC} 04$, in order not to charge the figure unnecessarily here.
} 


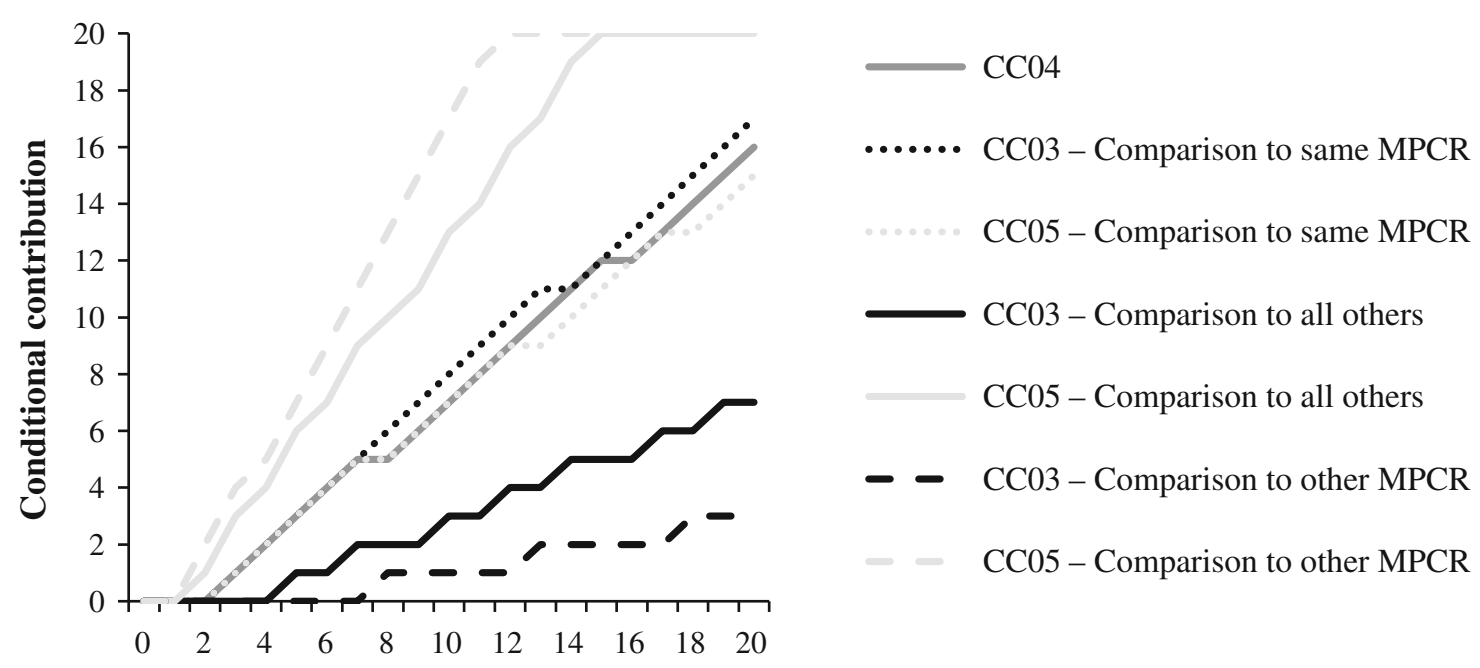

Average contribution of other group members

Fig. 2 Optimal conditional contributions-ERC model $\left(\right.$ with $\left.\vartheta_{i}=2000\right)$

Predictions of the FS and ERC model allow us to formulate three hypotheses. Both the FS and the ERC model predict that individuals may modify their contribution behavior when heterogeneity of returns from the public good is introduced. The FS model as well as the ERC model predict that participants make higher conditional contributions in $\mathrm{CC} 05$ than in $\mathrm{CC} 04$ than in $\mathrm{CC} 03$.

Hypothesis 1 (MPCR effect) Compared to the homogeneous MPCR of 0.4, the average of conditional contributions are higher in $\mathrm{CC} 05$ and lower in $\mathrm{CC} 03$.

Further, both models suggest that for sufficiently strong inequity considerations heterogeneity affects conditional contributions on average negatively.

Hypothesis 2 (Heterogeneity effect) The average of conditional contributions in CC05 and $\mathrm{CCO} 3$ is lower than the conditional contributions in the homogeneous case with an MPCR of 0.4 .

The predictions with respect to conditional contributions under uncertainty about the own MPCR differ between the two models: the FS model predicts that subjects strongly reduce conditional contributions whereas the ERC model predicts that contributions in $\mathrm{CCu0305}$ are only weakly smaller than contributions in $\mathrm{CCO} 4$ and do not differ by more than one point. Therefore, we will take the experimental results as a guide to the understanding of the effect of uncertainty in addition to heterogeneity.

Because different players may perceive the game differently, we also derived predictions for the FS and ERC model for subjects who compare only to a specific reference group. Both models suggest that conditional contributions should strongly react to heterogeneity if subjects compare themselves only to group members with the other MPCR, and that reactions to heterogeneity are rather weak if subjects compare only to group members with the same MPCR.

Hypothesis 3 (Type-specific reactions) Conditional cooperators' reactions to heterogeneity differ such that one fraction of conditional cooperators strongly increase conditional contributions in CC05 and strongly decrease conditional contributions in CC03 whereas another fraction reacts only weakly to heterogeneity in returns. 


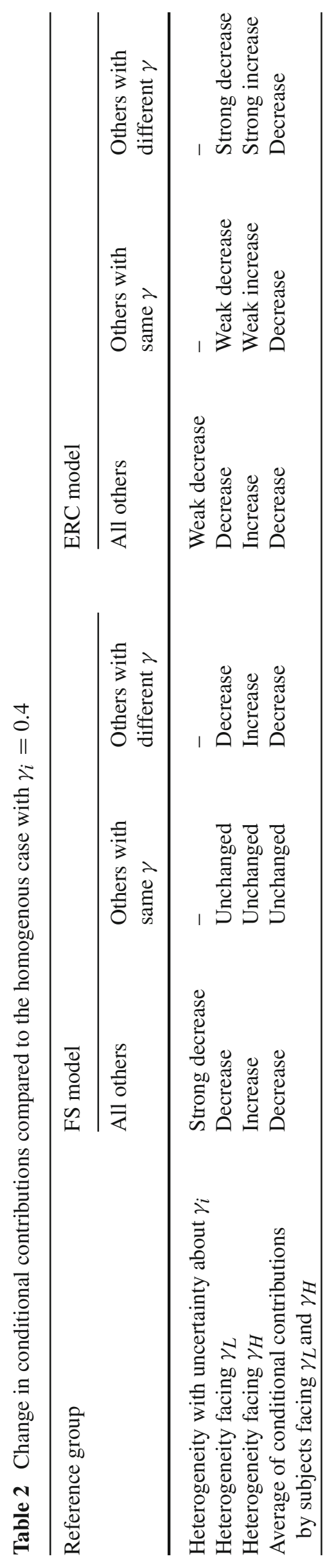


Fig. 3 Average unconditional contributions in UC games

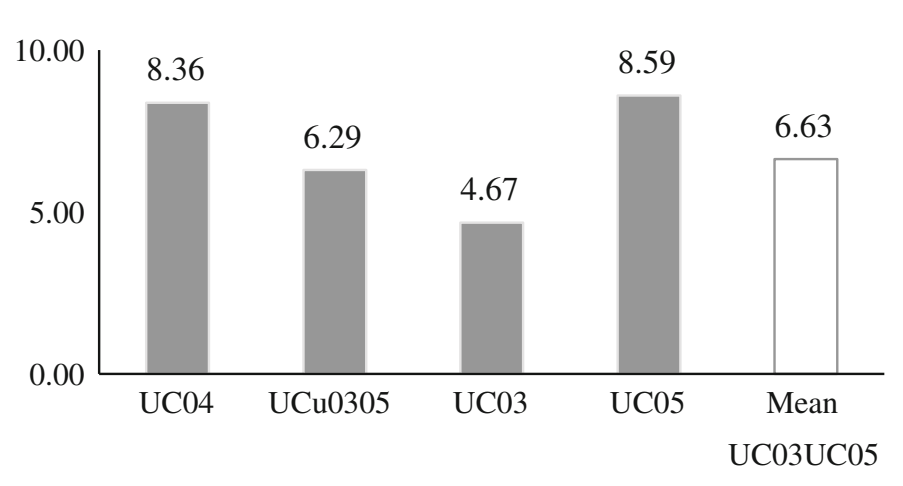

$(\mathrm{N}=228$, endowment $=20)$

\section{Results}

\subsection{Results from unconditional cooperation games (UC games)}

Figure 3 presents average unconditional contributions in the UC games as well as the mean of UC03 and UC05 as an additional benchmark. We observe significantly higher contributions to the public good when MPCRs are homogeneous rather than heterogeneous, irrespective of uncertainty (Wilcoxon signed-rank test: UC04 vs. UCu0305, $z=5.526, p<0.001$ and UC04 vs. MeanUC03UC05, $z=3.894, p<0.001) .{ }^{12}$ Subjects on average contribute positive amounts even under uncertainty about the own MPCR.

The introduction of uncertainty in addition to heterogeneity only slightly lowers subjects' contributions (Wilcoxon signed-rank test: UCu0305 vs. MeanUC03UC05, $z=2.316, p=0.021)$. Average unconditional contributions are lower in UC03 than in UC04 (Wilcoxon signed-rank test: $z=8.094, p<0.001$ ) and weakly higher in UC05 than in UC04 (Wilcoxon signed-rank test: $z=1.775, p=0.076$ ). Nevertheless, the decrease of contributions between UC04 and UC03 (Wilcoxon signed-rank test: $z=3.894, p<0.001)$ is much stronger than the increase of contributions between UC04 and UC05. Isaac and Walker (1998) showed that MPCRs and contributions are positively related in homogeneous environments. We cannot completely confirm this finding for heterogeneous environments. We find that lower returns induce a decrease of contributions when MPCRs are heterogeneous; we only observe a weak increase in contributions with high MPCRs in the heterogeneous environment. Thus the (positive) effect of the value of the MPCR seems to interact with the (negative) effect of heterogeneity of group members' MPCRs.

The results on unconditional contributions give the global effect of heterogeneity in returns on average contributions to the public good. However, the decrease in unconditional contributions might be driven by pessimistic beliefs about other group members' contributions. More precisely, conditional cooperators may underestimate other players' contributions in the heterogeneous case because they are unclear about what contribution norms other players follow. In public goods games, players may follow at least three focal contribution norms: equality in outcomes, equality in con-

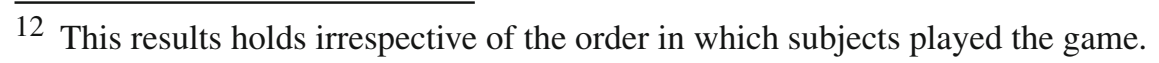


Fig. 4 Average conditional contributions $(\mathrm{N}=228)$

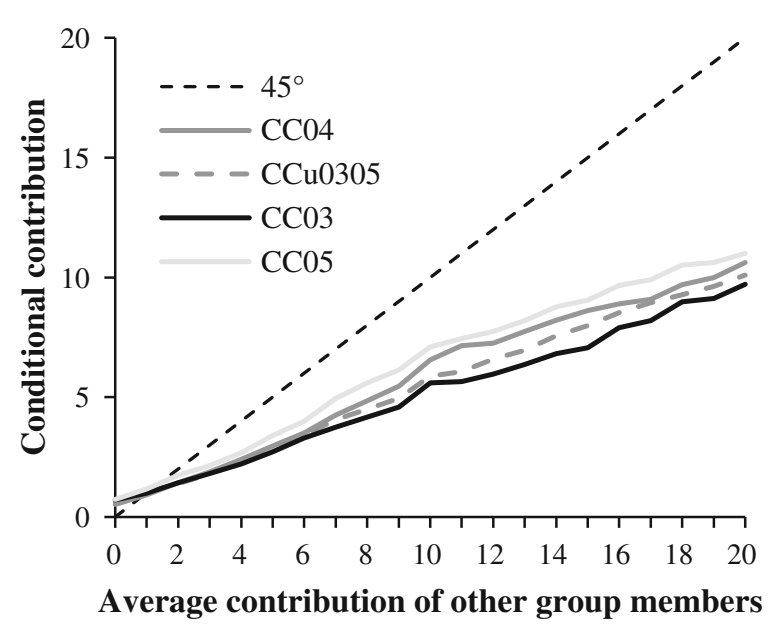

tributions or fairness with respect to individual ability. ${ }^{13}$ With homogeneous marginal returns, these norms result in the same predictions for contribution behavior (i.e. same contributions). With heterogeneity in returns these norms predict different contribution behavior. The results on conditional contributions will show whether this idea is reasonable because conditional contributions are independent of beliefs about group members' average contributions.

\subsection{Results from conditional cooperation games (CC games)}

Figure 4 shows average conditional contributions for all subjects in all treatments. On average, conditional contributions in CC04 are 5.81, in CC05 6.31 and in CC03 5.10 (unless otherwise specified, all averages are significantly different from another at the $1 \%$ level according to Wilcoxon signed rank tests). Subjects (on average) increase their conditional contributions in CC05 compared to CC04 whereas they decrease conditional contributions in $\mathrm{CC} 03$. The average of conditional contributions in $\mathrm{CC} 03$ and $\mathrm{CC} 05$ is equal to 5.70, which is not significantly different from the average of conditional contributions in $\mathrm{CC} 04$. Hence, introducing heterogeneity in MPCRs does not modify the average of conditional contributions. Regarding uncertainty, the average of conditional contributions in $\mathrm{CCu} 0305$ is equal to 5.45 and is significantly lower than the average of conditional contributions in CC03 and CC05 (significant at the $10 \%$ level), and in CC04. Therefore, uncertainty about MPCRs adds a negative impact of heterogeneity on the average of conditional contributions.

To investigate whether subjects adjust conditional contributions by changing their conditional contribution for every given average contribution level (i.e. they adjust the slope of their contribution schedule) or whether subjects simply become more or less generous when heterogeneity is introduced (i.e. they shift their schedule), we regress subjects' conditional contributions on the average contribution by their group members for all subjects and treatments in model (1) of Table 3.

\footnotetext{
13 For a more detailed discussion see also Konow (2003), Konow et al. (2009) and Reuben and Riedl (2013).
} 
Table 3 OLS regressions on conditional contributions

\begin{tabular}{|c|c|c|c|c|}
\hline \multirow{2}{*}{$\begin{array}{l}\text { Dependent variable } \\
\text { Conditional contribution }\end{array}$} & \multicolumn{2}{|l|}{ All subjects } & \multicolumn{2}{|c|}{ Conditional cooperators } \\
\hline & (1) & (2) & (3) & (4) \\
\hline $\mathrm{CC} 04$ & Ref. & Ref. & Ref. & Ref. \\
\hline $\mathrm{CCu} 0305$ & $\begin{array}{l}-0.048 \\
(0.108)\end{array}$ & $\begin{array}{l}-0.048 \\
(0.108)\end{array}$ & $\begin{array}{l}-0.009 \\
(0.110)\end{array}$ & $\begin{array}{l}-0.009 \\
(0.110)\end{array}$ \\
\hline MeanCC03CC05 & & $\begin{array}{l}0.134 \\
(0.121)\end{array}$ & & $\begin{array}{l}0.285^{* *} \\
(0.143)\end{array}$ \\
\hline $\mathrm{CC} 03$ & $\begin{array}{l}-0.044 \\
(0.151)\end{array}$ & & $\begin{array}{l}0.045 \\
(0.183)\end{array}$ & \\
\hline $\mathrm{CC} 05$ & $\begin{array}{l}0.311 * * \\
(0.134)\end{array}$ & & $\begin{array}{l}0.524 * * * \\
(0.150)\end{array}$ & \\
\hline $\begin{array}{l}\text { Average contribution of } \\
\text { other group members } \\
\text { (group average) }\end{array}$ & $(0.031)$ & $\begin{array}{l}0.523 * * * \\
(0.031)\end{array}$ & $(0.028)$ & $\begin{array}{l}0.816 * * * \\
(0.028)\end{array}$ \\
\hline Group average $\times \mathrm{CCu} 0305$ & $\begin{array}{l}-0.031 * \\
(0.016)\end{array}$ & $\begin{array}{l}-0.031 * \\
(0.016)\end{array}$ & $\begin{array}{l}-0.078 * * * \\
(0.020)\end{array}$ & $\begin{array}{l}-0.078 * * * \\
(0.020)\end{array}$ \\
\hline Group average $\times$ MeanCC03CC05 & & $-0.024(0.022)$ & & $-0.097 * * *(0.026)$ \\
\hline Group average $\times \mathrm{CC} 03$ & $\begin{array}{l}-0.067 * * * \\
(0.023)\end{array}$ & & $\begin{array}{l}-0.146^{* * * *} \\
(0.028)\end{array}$ & \\
\hline Group average $\times$ CC05 & $\begin{array}{l}0.019 \\
(0.024)\end{array}$ & & $\begin{array}{l}-0.048^{*} \\
(0.028)\end{array}$ & \\
\hline Constant & $\begin{array}{l}0.582 * * * \\
(0.208)\end{array}$ & $\begin{array}{l}0.582 * * * \\
(0.208)\end{array}$ & $\begin{array}{l}-0.205 \\
(0.193)\end{array}$ & $\begin{array}{l}-0.205 \\
(0.193)\end{array}$ \\
\hline Observations & 19,152 & 14,364 & 12,096 & 9,072 \\
\hline \# Clusters & 228 & 228 & 144 & 144 \\
\hline $\mathrm{R}^{2}$ & 0.225 & 0.229 & 0.489 & 0.516 \\
\hline
\end{tabular}

We ran additional regressions controlling for order effects. We find that subjects become less generous as the experiment progresses. Considering model (1), subjects who first face the heterogeneous case contribute in $\mathrm{CCO} 4$ about the average of their conditional contribution in $\mathrm{CCO} 3$ and CC05. Subjects who face the homogeneous situation first contribute slightly less than the average of their conditional contribution in CC03 and CC05. For conditional cooperators - models (3) and (4)—we find qualitatively similar results for both orders

Robust standard errors in parentheses

$* * * p<0.01, * * p<0.05, * p<0.1$

We first observe that if MPCRs are homogeneous, an increase of the average contributions of group members by one point will lead to an increase in conditional contribution by 0.523 . The results show that in $\mathrm{CC} 03$ subjects decrease their slope significantly by 0.067 whereas they do not significantly increase their slope in CC05. Instead, they behave more generously in CC05 by shifting up the intercept of their schedule by 0.311 . Result 1 summarizes results regarding the MPCR effect. Hypothesis 1 cannot be rejected. 
Result 1 On average, conditional contributions are higher in $\mathrm{CC} 05$ and lower in $\mathrm{CC} 03$ compared to the homogeneous MPCR of 0.4 .

In order to test hypothesis 2 , we consider the average conditional contribution of $\mathrm{CC} 03$ and $\mathrm{CC} 05$ for each individual subject and each average of group members' contributions to measure the aggregate effect of the introduction of heterogeneity in returns in model (2). Interestingly, heterogeneity without uncertainty does not significantly affect neither the slope of players' conditional contributions nor their generosity level. This suggests that heterogeneity in particular affects subjects' beliefs about others' contributions. We summarize findings related to the heterogeneity effect in result 2.

Result 2 On average, conditional contributions of subjects with heterogeneous MPCRs do not significantly differ from conditional contributions with homogenous MPCRs.

While there is no aggregate effect of heterogeneity without uncertainty on conditional contributions the joint-effect of uncertainty and heterogeneity from MPCRs on the slope of contribution schedules is significantly negative. The generosity of players is not significantly affected. Uncertainty additionally to heterogeneity decreases a little the generosity of players ( $F$ test for equality of coefficients ' $\mathrm{CCu} 0305$ ' and 'MeanCC03CC05': $p=0.078$ ) but has no significant effect on the slope of contribution schedules ( $F$ test for equality of coefficients 'Group average $\times \mathrm{CCu} 0305$ ' and 'Group average $\times$ MeanCC03CC05': $p=0.681$ ). We summarize these findings in Result 3.

Result 3 Uncertainty in MPCRs reduces the average of conditional contributions compared to homogenous MPCRs and heterogeneous MPCRs without uncertainty.

This result contradicts the Fehr-Schmidt model which predicts (due to the linearity of the model) a sharp decrease in contributions. Further contributions in CC04 are by more than 1 point higher than in $\mathrm{CCu} 0305$ for almost all group average contributions larger than $10 .{ }^{14}$ Hence also the predictions of the ERC model are not well reflected by the data.

\subsection{Type-specific reactions}

To study individual and type-specific reactions, we classify subjects based on their behavior in $\mathrm{CC} 04$ for the subsequent analysis. We define preference types according to the procedure introduced by Fischbacher et al. (2001): selfish subjects are subjects who always contribute zero to the public good; conditionally cooperative subjects are subjects who monotonically increase their contribution to the public good as the average contribution of other group members increases or whose contributions are significantly positively correlated to the average contribution of other group members. The last type of subject shows a hump-shaped contribution pattern, i.e. these subjects' contributions are increasing in the average contribution of other group members until a specific value and then decrease in it.

$\overline{14}$ The exceptions are group averages of 12 and 17. 


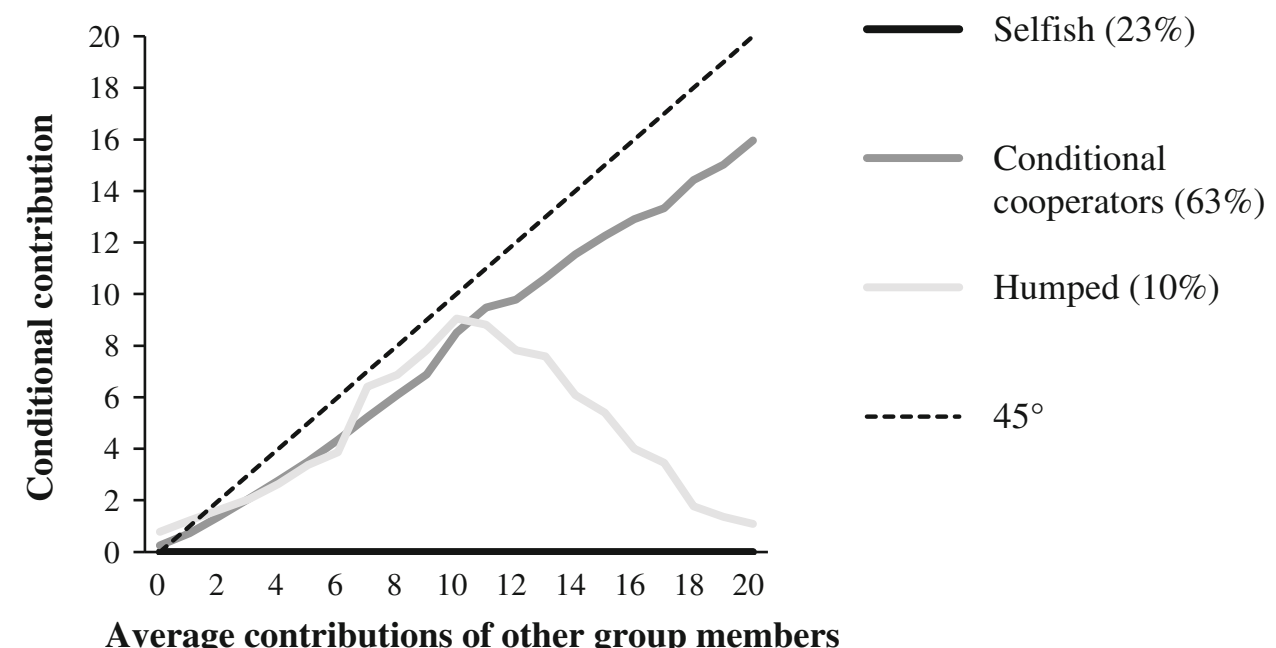

Fig. 5 Average of conditional contributions in CC04 by type

Over the 228 participants, we observe $23 \%$ of selfish subjects, $10 \%$ show a humpedshaped pattern and $63 \%$ are conditional cooperators. Only $4 \%$ of the participants do not fit in any of these categories. ${ }^{15}$ The contribution of each type for all potential average contributions of other group members in CC04 is depicted in Fig. 5. Subjects with a humped-shaped pattern and subjects who do not follow a specific strategy are few (in total they represent $14 \%$ of the subjects). We briefly report the results for subjects categorized as humped-shaped and selfish but concentrate our analysis on conditionally cooperative subjects.

\subsubsection{Selfish subjects and subjects with a humped-shaped contribution schedule}

Almost all subjects who are classified as selfish in CC04 contribute zero to the public good for any average contribution of other group members in CC03, CC05 and $\mathrm{CCu} 0305$. Thus heterogeneity does not significantly affect contribution behavior of these subjects. ${ }^{16}$ Conditional contribution schedules with humped-shaped patterns are rare (22 subjects out of 228). Subjects with such schedules contribute on average 4.43 in $\mathrm{CC} 04$. Average contributions are higher when heterogeneity is introduced, weakly in CC03 (5.66, Wilcoxon signed-rank tests, $p=0.0998$ ) and strongly in CC05 (7.02, $p=0.0002$ ). Changes in average contributions are mainly caused by eight subjects, who show a humped-shaped pattern in $\mathrm{CC} 04$ but are conditionally cooperative either in $\mathrm{CC} 03, \mathrm{CC} 05$ or both. Subjects showing a humped-shaped pattern in all three situations (CC04, CC03, CC05, n=11) are only weakly affected by heterogeneity.

\footnotetext{
15 As a comparison, Fischbacher et al. (2001) find about one third of subjects classified as free riders whereas about $50 \%$ are conditionally cooperative.

16 Six out of 52 as selfish classified subjects contribute more than zero in UC03, UC05. Among them four who slightly increase contributions in both UC03 and UC05 and two who only increase their contributions in UC05.
} 
Fig. 6 Average of conditional contributions by conditional cooperators

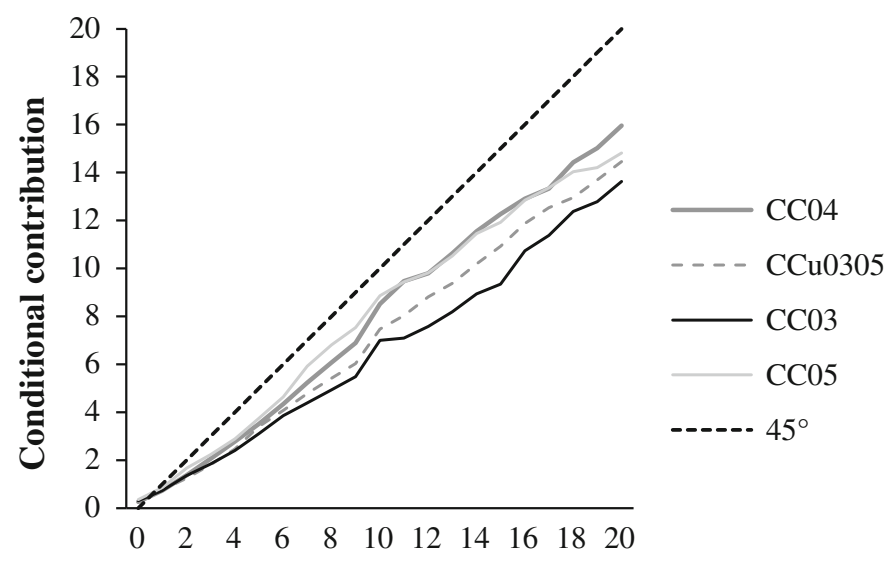

Average contributions of other group members

\subsubsection{Conditional cooperators-at the aggregate level}

Figure 6 presents conditional contributions for subjects classified in $\mathrm{CC} 04$ as conditional cooperators. As for the whole sample, the average of conditional contributions by conditional cooperators is higher in $\mathrm{CC} 05$ than in $\mathrm{CC} 04$ than in $\mathrm{CC03}$, and lower in $\mathrm{CCu} 0305$ than in $\mathrm{CC} 04$. Average conditional contributions in $\mathrm{CCO} 4$ are 7.96, in $\mathrm{CC} 05$ 8.00, in $\mathrm{CCO} 3$ 6.54, in $\mathrm{CCu0305} 7.17$ and the average of $\mathrm{CC} 03$ and $\mathrm{CC} 05$ is 7.27. All these averages are significantly different at the $5 \%$ level (using paired two-sided $t$ tests) except the difference between $\mathrm{CC} 04$ and $\mathrm{CC} 05$ as well as between $\mathrm{CCu} 0305$ and the average of $\mathrm{CC} 03$ and $\mathrm{CC} 05 .{ }^{17}$ In contrast to the reactions of the full sample, conditional cooperators (on average) slightly reduce their contributions when heterogeneity is introduced. However, the decrease in average conditional contributions of conditional cooperators is small (about 9\%) compared to the decrease in average unconditional contributions (about 20\%) when heterogeneity is introduced. Also, uncertainty about the own MPCR does not (additionally) affect conditional contributions by conditional cooperators on average.

To study conditional contributions by conditional cooperators in more detail, we rerun our regressions models for conditional cooperators separately [see Table 3, models (3) and (4)]. The regressions show that if MPCRs are homogeneous, an increase of the average contributions of group members by one point will lead to an increase in conditional contribution by 0.816 . According to model (3), when the own MPCR is certain, the positive effect of group members' average contribution is significantly smaller in $\mathrm{CCO} 3$ and $\mathrm{CC} 05$ than in CC04 and is also smaller in $\mathrm{CC} 03$ than in $\mathrm{CC0}$ ( $F$ tests: $p<0.001)$. Besides, a high MPCR makes conditional cooperators on average more generous $(+0.524$ points irrespective of the group average compared to $\mathrm{CC} 04)$ but a low MPCR does not shift their conditional contribution schedule. Model (4) shows that the slope of contribution schedules is significantly lower when heterogeneity of MPCRs is introduced, with and without uncertainty about the own MPCR (partly as a result of the higher intercept in CC05). Regarding the additional effect of uncertainty to heterogeneity in returns, we find that subjects are more generous when they know

$\overline{17}$ The significance can be confirmed with Wilcoxon signed-rank tests. 
Table 4 Classification of conditional cooperators

\begin{tabular}{lccccc}
\hline & Share (\%) & Average Diff03 & Average Diff05 & SD Diff03 & SD Diff05 \\
\hline $\begin{array}{l}\text { Comparison with the } \\
\quad \text { same MPCR subject }\end{array}$ & 31.9 & 0.06 & 0.01 & 0.29 & 0.45 \\
$\begin{array}{l}\text { Comparison to all others } \\
\quad \text { \& to opp. MPCR }\end{array}$ & 17.4 & $-1.60 * * *$ & $0.59 * * *$ & 0.65 & 0.61 \\
Comparison to 05 subject(s) & 12.5 & $-5.39 * * *$ & 0.76 & 2.07 & 1.55 \\
Comparison to 03 subject(s) & 14.6 & $0.77 * * *$ & $1.75^{* * *}$ & 1.04 & 0.83 \\
Heterogeneity averse & 16.7 & $-4.95 * * *$ & $-4.70^{* * * *}$ & 2.56 & 2.78 \\
Heterogeneity lover & 6.9 & $3.39 * * *$ & $5.28 * * *$ & 2.43 & 2.96 \\
\hline
\end{tabular}

Stars indicate whether the medians are significantly different from zero according to Wilcoxon sign rank tests, with $* p$ value $<0.10,{ }^{* *} p$ value $<0.05$ and ${ }^{* * *} p$ value $<0.01$

their own MPCR ( $F$ test, $p=0.013$ ) but the slope coefficients do not significantly differ ( $F$ test, $p=0.359$ ). Overall, the regression results of the full sample are similar to the results for conditional cooperators. We only observe a different behavior when heterogeneity from returns without uncertainty is introduced.

\subsubsection{Conditional cooperators—at the individual level}

Hypothesis 3 suggests heterogeneous reactions to heterogeneity in MPCRs. To investigate hypothesis 3, we use the hierarchical cluster analysis of Ward (1963). This method is based on the minimization of the intra-group variance. At each step in the analysis, the union of every possible cluster pair is considered and the two clusters whose fusion results in minimum increase in variance are combined. To classify subjects, we use two variables reporting how subjects' conditional cooperation differs between CC04 and CC03 and between CC04 and CC05. The first variable is the average of each subject's difference in conditional contributions in CC03 and CC04. We call this variable Diff03 (Diff03 $=$ average of $\left(g_{i 03}-g_{i 04}\right)$. Diff03 being negative indicates that subjects' conditional cooperation is less perfect when the subject's MPCR equals 0.3 (with heterogeneity of MPCRs) than when it equals 0.4 (with homogeneity). To compute the second variable, we do the same but replace the low MPCR by the high MPCR. We name this second variable Diff05 $=g_{i 05}-g_{i 04}$. We report the results in Table 4.

On average, the difference between the average contribution of other group members and the conditional contribution of a conditional cooperator is equal to 2.04 in CC04. When there is heterogeneity in MPCRs, this value is equal to 3.45 if subjects receive the low MPCR, 2.00 if subjects receive the high MPCR and 2.83 if they do not know which MPCR they will receive. We have thus Diff03 $=-1.41$ and Diff05 $=0.04$. We identify 6 categories of subjects with the Ward's classification method. For each category of subjects, the average and standard deviation of Diff03 and Diff05 as well as the share of conditional cooperators it includes are presented in Table 4. From Ward's classification of conditional cooperators, we can infer whether behavior corresponds to the classifications suggested by theory. 
On the one hand, $31.9 \%$ of conditional cooperators behave as if they compare their payoff to the payoff of the other group member receiving the same MPCR. They do not significantly change their behavior as heterogeneity in MPCRs is introduced (Wilcoxon signed rank test, for Diff03: $p$ value $=0.65$, for Diff05: $p$ value $=0.34$ ). ${ }^{18}$ On the other hand, $17.4 \%$ of conditional cooperators behave as if they compare their payoff to the average payoff of all other group members, or to the two group members having the opposite MPCR. These subjects modify their conditional contributions to the public goods as heterogeneity of MPCRs is introduced: they significantly decrease their contributions to the public good when they receive the low MPCR and increase it when they receive the high MPCR (Wilcoxon signed rank test for difference with 0 , $p<0.01$ for Diff03 and Diff05). ${ }^{19}$ Hence, we cannot reject hypothesis 3 .

Result 4 A positive share of conditional cooperators do not modify their conditional contributions as heterogeneity in MPCRs is introduced while another positive share increase them in $\mathrm{CCO} 5$ and decrease them in $\mathrm{CCO} 3$ compared to $\mathrm{CC} 04$.

Ward's classification yields two further categories, in which behavior corresponds roughly to the theoretical predictions for subjects who compare their payoff only to payoffs of subjects with a specific MPCR of either 0.3 or 0.5 . According to the theoretical model, for subjects who only compare to others with an MPCR of 0.5 their conditional contributions in $\mathrm{CC} 04$ should be higher than in $\mathrm{CC} 03$ and higher than or equal to in CC05. Indeed, $12.5 \%$ of conditional cooperators roughly behave in this way (Wilcoxon signed rank test for difference with $0, p<0.01$ for Diff03 and $p=0.123$ for Diff05). Behavior of another $14.6 \%$ of conditional cooperators roughly coincides with the predictions for subjects who compare their payoff only to payoffs of members receiving an MPCR of 0.3. They significantly increase their contribution when they receive the high MPCR and slightly increase it when they receive the low MPCR (Wilcoxon signed rank test for difference with $0, p<0.01$ for Diff03 and Diff05).

The two last categories include subjects who are affected by the introduction of heterogeneity in MPCRs in the same way by both CC03 and CC05. We name $16.7 \%$ of our subjects "heterogeneity averse" because they significantly decrease their contribution when heterogeneity is introduced irrespective of their own MPCR (Wilcoxon signed rank test for difference with $0, p<0.01$ for Diff03 and Diff05). ${ }^{20}$ A minority

\footnotetext{
18 On theoretical grounds no reaction to heterogeneity in returns may also result from comparisons in contributions instead of final payoffs. However, in the experiment subjects do not observe individual contributions and may thus only match average contributions. It is also plausible that subjects facing first homogeneity and then heterogeneity stick to their behavior in the homogeneous treatment because they are missing information necessary to fulfill their fairness norms in the heterogeneous case (e.g. individual contributions). However, the data do not suggest such an order effect. The probability to be in the group that does not react to heterogeneity is not significantly different (Wilcoxon rank-sum test, $p=0.413$ ) and even lower when facing homogeneity first (0.184) compared to facing heterogeneity first $(0.228)$.

19 We cannot separate subjects comparing themselves to subjects with the opposite MPCR from subjects comparing to all others, because the theoretical predictions do not differ qualitatively.

20 Heterogeneity averse people are actually classified into two different clusters. Although average Diff03 and average Diff05 have the same sign in both clusters, the magnitude is different. We group these two clusters because for both Diff03 and Diff05 are strongly negative. Each cluster presents $8.3 \%$ of the population. In the first cluster, average Diff03 is -6.94 and average Diff05 is -6.86 while in the second cluster these values are respectively -2.97 and -2.54 .
} 
of $6.9 \%$ of subjects behaves "heterogeneity loving", i.e. they significantly increase their contribution in $\mathrm{CCO} 3$ and $\mathrm{CC} 05$ compared to $\mathrm{CCO} 4$ (Wilcoxon signed rank test for difference with $0, p<0.01$ for Diff03 and Diff05).

\section{Conclusion}

We investigated whether the introduction of heterogeneity and uncertainty in returns from public goods affects unconditional and conditional contribution behavior. Unconditional contributions depend on beliefs whereas conditional contributions are beliefindependent. A within-subject design allowed us further to analyze reactions to heterogeneity in MPCRs from the public good at the individual level. Based on the assumption that subjects may compare to different reference groups, we hypothesized that individuals react differently to heterogeneity in returns.

The results show that, at the aggregate level, heterogeneity in MPCRs from the public good reduces unconditional contributions significantly, regardless of whether the own MPCR from the public good was certain or uncertain. However, conditional contributions are less strongly affected by heterogeneity, suggesting that negative effects of heterogeneous environments may in particular result from more pessimistic beliefs about others' contribution behavior caused by a missing common contribution norm. The fact that it is not clear which contribution norm is common (equality in payoffs, equality in contributions or proportionality with respect to marginal returns) may cause a reduction in players' unconditional contributions in our one shot game without punishment. However, Reuben and Riedl (2013) have recently shown that repeated interactions and punishment possibilities may clarify a common contribution norm and may mitigate the negative effects of heterogeneity on contributions.

Decomposing our results on conditional contributions shows that reactions to heterogeneity in returns are heterogeneous. Differences in reactions are systematic. Heterogeneity does not affect selfish subjects' behavior significantly. Conditional cooperators' reactions are mixed. We detect around one third of conditional cooperators who do not react to heterogeneity in MPCRs. $17 \%$ of conditional cooperators decrease their contributions when they receive the low MPCR and increase it when they receive the high MPCR. Additionally, some conditional cooperators mainly react to only high or low returns while others have the same reaction regarding both returns when heterogeneity is introduced. A substantial part of this variation can be explained by accounting for different reference groups subjects may compare to. The decomposition of results on conditional contributions yields an important insight: heterogeneity decreases conditional contributions mainly for two types of conditional cooperators. The first type dislikes heterogeneity in general. The second type behaves as if comparing only to group members with higher returns from the public good.

At this point it is worthwhile to note that our conditional contribution treatments reflect a situation in which people are aware that they may benefit equally or unequally from the public good but only observe aggregate contributions. For example, people may observe the general air quality in a city, be aware that those living in the center benefit most from it, but do not observe individual pollution levels and thus are not aware of individual contributions. Individuals who refer to a specific reference group's 
contributions need to conjecture these contributions. Because the conjecture is uncertain and people are aware of this uncertainty they may also be tempted to refrain from applying their fairness norm and instead use a fairness norm which requires less information (e.g. simply matching the average contribution). Thus, the individual reactions to heterogeneity observed in our experiment may not only reflect behavioral changes with respect to specific reference groups but also changes in the fairness norms of our subjects.

Our investigation provides insights which may on the one hand enrich theoretical models of individual behavior and on the other hand help to develop policies which may increase contributions to public goods when returns from the public goods are heterogeneous. With respect to behavioral theories the individual level analysis is particularly interesting. Although subjects' contribution behavior in the homogeneous case may be explained by inequality aversion as modeled by the FS and the ERC models, the reactions to heterogeneity differ and cannot fully be explained by these models. In particular, it seems as if inequity averse subjects differ in the way they compare their payoff to the payoff of other group members. The economic literature already shows that people compare their payoff to a reference payoff level they have in mind and differ in this reference level. Depending on this reference level, people feel more or less happy with the same payoff (see for instance Clark and Oswald 1996). Our results suggest that reference groups also play a crucial role for other regarding preferences. While previous research has shown the existence of three main types of subjects: selfish, conditionally cooperative and humped-shaped subjects. Our experiment additionally suggests that conditional cooperators differ in their reference group: some compare their payoffs only to people who look like them, or only to people who are different; some instead compare their payoffs exclusively to advantaged people and others to disadvantaged ones. In order to analyze cooperation in a society with inequality, it may be worthwhile to develop behavioral theories including heterogeneity in reference groups. A path for further research would be the exploration of inequity aversion or reciprocity models including an underlying reasoning for the choice of a specific reference group.

With respect to policy recommendations it is particularly interesting that heterogeneity reduces unconditional contributions but does only marginally affect conditional contributions. On the one hand the reduction in unconditional contributions points out that policies may aim at reducing heterogeneity in returns to public goods through compensations. A drawback of compensations is that they require the policy maker to be able to identify returns from public goods for different individuals. Hence compensations might be difficult to be implemented in practice. On the other hand, the fact that conditional contributions are only marginally affected suggests that belief management through communication campaigns is even more important in the situation of heterogeneity. While pessimistic beliefs may be intrinsic to individuals or depend on past experience, it has been shown that beliefs about others' cooperation may be affected in public goods games by providing information about the level of cooperation in other unrelated groups (Engel et al. 2011). There are several ways how to influence beliefs about others' contribution behavior. One may generally de-emphasize heterogeneity in returns, emphasize the fact that there are multiple public goods which may counterbalance heterogeneity in returns or establish a common 
contribution norm (see also Reuben and Riedl 2013). De-emphasizing heterogeneity without deceiving the public is difficult but emphasizing the existences of multiple public goods or establishing common contribution norms seems feasible.

Acknowledgments We thank Kate Bendrick, Lisa Bruttel, Gerald Eisenkopf, Pascal Sulser, Verena Utikal and Irenaeus Wolff as well as the participants of the ESA Meeting 2010 in Copenhagen, the THEEM workshop 2010 in Kreuzlingen and the ASFEE Conference 2011 in Fort-de-France as well as two anonymous referees for their helpful comments. Financial support is acknowledged from the Swiss Federal Office of Energy.

\section{References}

Anderson LR (2001) Public choice as an experimental science. In: Shughart W, Razzolinis L (eds) The Elgar companion to public choice. Edward Elgar, Cheltenham, pp 497-511

Bagnoli M, McKee M (1991) Voluntary contribution games: efficient private provision of public goods. Econ Inq 29(2):351-366

Bohm P (1972) Estimating demand for public goods: an experiment. Eur Econ Rev 3(2):111-130

Bolton GE, Ockenfels A (2000) ERC: a theory of equity, reciprocity, and competition. Am Econ Rev 90(1):166-193

Carpenter J, Bowles S, Gintis H, Hwang SH (2009) Strong reciprocity and team production: theory and evidence. J Econ Behav Org 71(2):221-232

Chan K, Mestelman S, Moir R, Muller R (1999) Heterogeneity and the voluntary provision of public goods. Exp Econ 2(1):5-30

Chaudhuri A (2011) Sustaining cooperation in laboratory public goods experiments: a selective survey of the literature. Exp Econ 14(1):47-83

Cheung S (2011) New insights into conditional cooperation and punishment from a strategy method experiment. IZA discussion paper no. 5689.

Clark AE, Oswald AJ (1996) Satisfaction and comparison income. J Public Econ 61(3):359-381

Dickinson D (1998) The voluntary contributions mechanism with uncertain group payoffs. J Econ Behav Org 35(4):517-533

Dufwenberg M, Kirchsteiger G (2004) A theory of sequential reciprocity. Games Econ Behav 47(2):268298

Engel C, Kube S, Kurschilgen M (2011) Can we manage first impressions in cooperation problems? An experimental study on "Broken (and Fixed) Windows". Working paper series of the Max Planck Institute for Research on Collective Goods

Falk A, Fischbacher U (2006) A theory of reciprocity. Games Econ Behav 54(2):293-315

Falkinger J, Fehr E, Gächter S, Winter-Ebmer R (2000) A simple mechanism for the efficient provision of public goods: experimental evidence. Am Econ Rev 90(1):247-264

Fehr E, Schmidt KM (1999) A theory of fairness, competition, and cooperation. Q J Econ 114(3):817-868

Fellner G, Iida Y, Kröger S, Seki E (2011) Heterogeneous productivities in public good production-An experimental study. IZA Bonn, Working Paper No. 5556

Fischbacher U (2007) z-Tree: Zurich toolbox for ready-made economic experiments. Exp Econ 10(2):171178

Fischbacher U, Gächter S, Fehr E (2001) Are people conditionally cooperative? Evidence from a public goods experiment. Econ Lett 71(3):397-404

Fisher J, Isaac R, Schatzberg J, Walker J (1995) Heterogenous demand for public goods: behavior in the voluntary contributions mechanism. Public Choice 85(3):249-266

Gächter S (2007) Conditional cooperation: behavioral regularities from the lab and the field and their policy implications. In: Frey B, Stutzers A (eds) Economics and psychology: a promising new cross-disciplinary field. MIT Press, Cambridge

Gangadharan L, Nemes V (2009) Experimental analysis of risk and uncertainty in provisioning private and public goods. Econ Inq 47(1):146-164

Greiner B (2004) An online recruitment system for economic experiments. Forschung und wissenschaftliches Rechnen GWDG Bericht 63, K. Kremer and V. Machos, Göttingen, pp 79-93 
Isaac R, Walker J (1988) Group size effects in public goods provision: the voluntary contributions mechanism. Q J Econ 103(1):179-199

Konow J (2003) Which is the fairest one of all? A positive analysis of justice theories. J Econ Lit 41(4):11881239

Konow J, Saijo T, Akai K (2009) Morals and mores? Experimental evidence on equity and equality. Working paper, Loyola Marymount University

Ledyard JO (1995) Public goods: a survey of experimental research. In: Roth AE, Kagels JH (eds) The handbook of experimental economics. Princeton University Press, Princetown, pp 111-181

Levati M, Morone A, Fiore A (2009) Voluntary contributions with imperfect information: an experimental study. Public Choice 138(1):199-216

Marwell G, Ames R (1980) Experiments on the provision of public goods. II. Provision points, stakes, experience, and the free-rider problem. Am J Sociol 85(4):926-937

Nikiforakis N, Noussair CN, Wilkening T (2012) Normative conflict and feuds: the limits of selfenforcement. J Public Econ 96(9-10):797-807

Rabin M (1993) Incorporating fairness into game - theory and economics. Am Econ Rev 83(5):1281-1302

Reuben E, Riedl A (2009) Public goods provision and sanctioning in privileged groups. J Confl Resolut 53(1):72

Reuben E, Riedl A (2013) Enforcement of contribution norms in public good games with heterogeneous populations. Games Econ Behav 77(1):122-137

Selten R (1967) Die Strategiemethode zur Erforschung des eingeschränkt rationalen Verhaltens im Rahmen eines Oligopolexperimentes. In: Sauermanns H (ed) Beiträge zur experimentellen Wirtschaftsforschung, Tübingen, pp 136-168

Tan F (2008) Punishment in a linear public good game with productivity heterogeneity. De Econ 156(3):269_ 293

Ward JH (1963) Hierachical grouping to optimize an objective function. J Am Stat Assoc 58:236-244

Wolff I (2013) Cooperation when player types are common knowledge. Working Paper

Zelmer J (2003) Linear public goods experiments: a meta-analysis. Exp Econ 6(3):299-310 